\title{
Instrumental with, locatum with and the argument/adjunct distinction ${ }^{1}$
}

\author{
Lilia Rissman \\ J ohns Hopkins University
}

\section{Introduction}

The majority of thematic roles fit fairly cleanly into the argument or adjunct category: agents and patients, for example, typically pattern as arguments, whereas temporal and locative expressions typically pattern as adjuncts. This paper explores the argumenthood properties of two event participants that are not so neatly categorized: instruments, as in (1), and locata, as in (2):

(1) J ill stuffed the cabbage with a spoon.

(2) J ill stuffed the cabbage with rice.

I ask whether instruments and locata pattern as arguments or adjuncts, and how cleanly they fit into these categories. Applying a variety of semantic and syntactic diagnostics, I argue that locata are best classified as arguments and instruments as adjuncts, and that argumenthood is gradient rather than categorical.

\section{Previous analyses}

Previous researchers have proposed at least three different ways of classifying instruments and locata into argument and adjunct categories. Consistent with my analysis, Levin \& Rappaport (1988) and J ackendoff (1990) propose that instruments are adjuncts and locata arguments. In contrast, Koenig, Mauner, Bienvenue \& Conklin (2008) argue that instruments may be arguments or adjuncts depending on the properties of the verbal head. Although they analyze locata as instances of the instrumental role, locata would pattern as arguments under their analysis. Schutze (1996) does not discuss locata but argues that instruments are arguments. I will consider some of the reasons for these classifications in my subsequent discussion of semantic and syntactic diagnostics of argumenthood.

\section{Semantic diagnostics of argumenthood}

\subsection{Restricted range of heads}

Schutze (1996) argues that arguments appear with a more restricted range of heads than adjuncts, as the contrast in (3) shows:

a. a man/ woman/ dog/ Muppet/scarecrow with gray hair

b. a member $/ * \mathrm{dog} / *$ Muppet $/ *$ scarecrow of Parliament

\footnotetext{
1 Thank you to Kyle Rawlins, Barbara Landau, J ennifer Culbertson, Ozge Gurcanli, and members of the Semantics and Language and Cognition Labs for help with this work. Thank you also to all survey participants. This work was supported by an NSF IGERT grant.
} 
The PP of Parliament would thus be an argument and with gray hair an adjunct. I observe that locata can only appear with a small class of "putting" verbs, such as spray, smear, fill and cover (I will refer to these verbs as "locatum verbs"):
a. I sprinkled the cake with coconut.
b. *I placed the table with books.
c. *I broke the floor with glass.

In contrast, instruments can modify any non-stative verb:
a. I charmed my boss with pleasantries.
b. I have to walk with a cane.
c. She blinded me with science.

Locata thus pattern as arguments and instruments as adjuncts on this diagnostic.

\subsection{Semantic obligatoriness}

In Koenig, Mauner \& Bienvenue's 2003 proposal, participants that are mandatory parts of the event that a verb describes are semantic arguments of that verb. Locata behave as arguments here because the existence of a locatum is always entailed by a locatum verb:

(6) a. Martha frosted the cake. (understood: with X)

b. Jill filled the vase. (with Y)

c. Chloe loaded the truck. (with Z)

Koenig et al. (2008) argue that the existence of an instrument is entailed for some verbs:

(7) a. Martha sliced the turkey. (understood: with X)

b. J ill chopped the onions. (with Y)

c. Chloe groomed the pony. (with Z)
a. Chloe put on her shoes.
b. Martha ran to the store.
c. J ill made a snow angel.

The verbs in (8) do not entail the presence of an instrument. Given this contrast, Koenig et al. (2008) propose that for a relatively small set of verbs, instruments compose with the verb as arguments, whereas in the general case instruments compose as adjuncts. An alternative possibility is that the contrast between (7) and (8) is evidence for gradience of argumenthood: given an argument/ adjunct spectrum, instruments could fall on the adjunct end while occupying a more medial position than most adjuncts.

\subsection{Dependence on head for interpretation}

Dowty (2003) discusses the contrast in (9): 
a. Mary walks to work.

b. Mary rented the apartment to J ohn.

He argues that because we can understand the semantic contribution of the PP independently from the meaning of the head in (9a) but not (9b), the PP is an adjunct in (9a) and an argument in (9b). For locata, the spatial relationship between the locatum and the location depends entirely on the verb:
a. I filled the cooler with ice.
$\rightarrow$ ice IN cooler
b. I covered the ground with a blanket.
c. I framed the painting with gold.
$\rightarrow$ blanket ON ground
$\rightarrow$ gold AROUND painting

Schutze (1996) observes that instrumental interpretation may be verb dependent, leading him to classify instruments as arguments:
a. I cut the bread with a knife.
$\rightarrow$ knife is causal intermediary
b. I ate the ice cream with a spoon.
$\rightarrow$ spoon is facilitating

Instrumental interpretation is not entirely encoded by the verbal head, however:
a. J ohn painted the ceiling with a roller.
b. J ohn painted the ceiling with a ladder.
c. J ohn painted the ceiling with only his left hand.

Properties of the verb, the DO and instrument as well as sentential context lead to three different interpretations of the role of the instrument in the painting event. Such flexibility is not possible for locatum interpretation, indicating that locata are arguments. I argue that the semi-head-dependence of instruments indicates that they are adjuncts, falling at a more medial position on an argument/ adjunct spectrum.

\section{Syntactic diagnostics of argumenthood}

I investigated whether the locata $\rightarrow$ argument; instrument $\rightarrow$ adjunct classification would emerge on syntactic diagnostics of argumenthood. Using a grammaticality judgment survey, I tested for contrasts on four syntactic diagnostics. 20 adult native English speakers rated the target sentences on a 1-10 scale, with 10 as best and 1 worst.

\subsection{Double-with diagnostic}

Given the contrast in (13), Levin \& Rappaport (1988) argue that locata are positioned internally to instruments and are therefore arguments:

(13) a. ? He loaded the cart with apples with a bucket. b. *He loaded the cart with a bucket with apples.

I tested the generality of this contrast using four verbs, load, stuff, fill and flood. Subjects prefered locatum-internal to instrument-internal sentences ( $M=5.6$ vs. $M=4.3$ ), 
consistent with the locata $\rightarrow$ argument; instrument $\rightarrow$ adjunct hypothesis.

\subsection{Adverb placement diagnostic}

Given the contrast between (14a-b), Randall (1992) suggested that locata are arguments, as adverbs cannot intervene between heads and complements:

a. ?She loaded the wagon quickly with hay.

b. She loaded the wagon quickly with a pitchfork.

c. She chopped the onions quickly with a knife.

I tested this contrast with locatum and non-locatum verbs as in (14c), using yesterday as well as quickly. Subjects did not distinguish between (a) and (b) forms ( $M=6.4$ vs. $\mathrm{M}=6.4$ ) but rated the (c) forms as better ( $\mathrm{M}=7.5)$. The contrast between (a) and (c) is preliminary evidence that the locatum has a more internal position than the instrument. Perhaps (b) was ranked lower than (c) for independent reasons; for example, subjects overall penalized sentences with overt instruments but implicit locata, as in 14b.

\subsection{Do-so replacement and weak wh-island extraction diagnostics}

My hypothesis predicts the following contrasts in (15) and (16):

(15) a. ?Bill loaded the truck with cartons and Don did so with trunks. b. Bill loaded the truck with a crane and Don did so with a forklift.

(16) a. With what kind of pumpkins did I ask how to load a wheelbarrow?

b. ?With what kind of ramp did I ask how to load a truck?

Subjects did not in fact distinguish these. Their responses to prototypical argument/ adjunct controls were not as expected, however, suggesting that these sentences are perhaps too unusual to elicit reliable judgments from untrained raters.

\section{Conclusion}

Using a range of semantic and syntactic diagnostics, I have argued that locata pattern as arguments and instruments as adjuncts. I interpret the non-prototypical behavior of instruments as evidence for gradience of argumenthood.

\section{References}

Dowty, D. (2003). The dual analysis of adjuncts/ complements in categorial grammar. In E. Lang, C. Maienborn \& C. FabriciusHansen (Eds.), Modifying adjuncts (pp. 33-66). Berlin: Mouton de Gruyter.

J ackendoff, R. (1990). Semantic structures. Cambridge, Mass.: MIT Press.

Koenig, J ., Mauner, G., \& Bienvenue, B. (2003). Arguments for adjuncts. Cognition, 2003, 89, 2, Sept, 89(2), 67-103.

Koenig, J ., Mauner, G., Bienvenue, B., \& Conklin, K. (2008). What with? the anatomy of a (proto)-role. J ournal of Semantics, 25(2), 175-220.

Levin, B. \& Rappaport, M.(1988). Nonevent-er nominals. Linguistics, 26(6), 1067-1083.

Randall, J. H. (1992). The catapult hypothesis: An approach to unlearning. In J. Weissenborn, H. Goodluck \& T. Roeper (Eds.),

Theoretical issues in language acquisition: Continuity and change in development (pp. 93-138). Hillsdale, NJ : Lawrence Erlbaum.

Schutze, C. T. (1996). PP attachment and argumenthood. MIT Working Papers in Linguistics, 26(Sept), 95-151. 